\title{
The impact of anxiety and catastrophizing on interleukin-6 responses to acute painful stress
}

This article was published in the following Dove Press journal: Journal of Pain Research

\author{
Asimina Lazaridou' \\ Marc O Martel' \\ Christine M Cahalan' \\ Marise C Cornelius' \\ Olivia Franceschelli' \\ Claudia M Campbell ${ }^{2}$ \\ Jennifer A Haythornthwaite ${ }^{2}$ \\ Michael Smith ${ }^{2}$ \\ Joseph Riley ${ }^{3}$ \\ Robert R Edwards' \\ 'Department of Anesthesiology, \\ Harvard Medical School, Brigham \& \\ Women's Hospital, Boston, MA, \\ USA; ${ }^{2}$ Department of Psychiatry \\ and Behavioral Sciences, School of \\ Medicine, Johns Hopkins University, \\ Baltimore, MD, USA; ${ }^{3}$ Department of \\ Community Dentistry and Behavioral \\ Science, College of Dentistry, \\ University of Florida, Gainesville, \\ FL, USA
}

Objective: To examine the influence of anxiety and pain-related catastrophizing on the time course of acute interleukin-6 (IL-6) responses to standardized noxious stimulation among patients with chronic pain.

Methods: Data were collected from 48 participants in the following demographically matched groups: patients with chronic pain $(n=36)$ and healthy controls $(n=12)$. Participants underwent a series of Quantitative Sensory Testing (QST) procedures assessing responses to mechanical and thermal stimuli during two separate visits, in a randomized order. One visit consisted of standard, moderately painful QST procedures, while the other visit involved nonpainful analogs to these testing procedures. Blood samples were taken at baseline, and then for up to 2 hours after QST in order to study the time course of IL-6 responses.

Results: Results of multilevel analyses revealed that IL- 6 responses increased across assessment time points in both visits $(p<0.001)$. While patients with chronic pain and healthy controls did not differ in the magnitude of IL-6 responses, psychological factors influenced IL- 6 trajectories only in the chronic pain group. Among patients, increases in catastrophizing over the course of the QST session were associated with elevated IL-6 responses only during the painful QST session $(p<0.05)$. When controlling for anxiety, results indicated that the main multilevel model among patients remained significant $(p<0.05)$.

Conclusion: Under specific conditions (eg, application of a painful stressor), catastrophizing may be associated with amplified proinflammatory responses in patients with persistent pain. These findings suggest that psychosocial interventions that reduce negative pain-related cognitions may benefit patients' inflammatory profiles.

Keywords: anxiety, catastrophizing, chronic pain, interleukin-6

\section{Introduction}

Chronic musculoskeletal pain is the leading cause of disability and impaired physical function in many parts of the world. ${ }^{1}$ Fibromyalgia (FM) ${ }^{2-4}$ and osteoarthritis (OA) ${ }^{5}$ are two relatively common musculoskeletal conditions that contribute to physical, occupational, and psychosocial disability; these disorders are characterized by persistent pain and hyperalgesia, fatigue and sleep disturbance, emotional distress, and impaired quality of life (QOL). Though FM and OA have distinct pathophysiologic mechanisms, proinflammatory cytokines such as interleukin-6 (IL-6) have been hypothesized to play a role in contributing to pain in both conditions. ${ }^{6-9}$ While FM is not considered an inflammatory disorder per se, van West and Maes ${ }^{10}$ suggested that inflammatory processes accompanied by changes in the neuroendocrine-immune system might underlie some of the symptomatology of FM, and a meta-analysis
Correspondence: Robert R Edwards Brigham \& Women's Hospital, Pain Management Center, 850 Boylston St, Chestnut Hill, Boston, MA 02467, USA

Tel +l 6177329486

Fax +I 6177329050

Email RREdwards@partners.org 
highlighted elevations in IL-6 as an important feature of FM pain. ${ }^{11}$ IL-6 also appears to play a role in generating or maintaining joint pain in OA, a condition characterized by persistent articular cartilage degeneration. In a recent study, IL-6 was elevated among OA patients compared to pain-free controls, and Doss et al suggested that variability in IL-6 levels within the OA group might characterize important patient subgroups. ${ }^{12}$ Other recent studies have also reported that serum IL-6 levels are higher in OA patients compared to controls. ${ }^{13}$ Of note, OA and FM patients matched for pain duration and pain intensity demonstrated similarly elevated proinflammatory cytokine profiles. ${ }^{9}$ In addition, the central nervous system interacts dynamically with the immune system to modulate inflammation through humoral and neural pathways. For example, activation of vagal afferents culminates in the release of acetylcholine that blocks cytokine production by cells expressing acetylcholine receptors. ${ }^{14}$ Such vagal and cholinergic modulation of inflammatory pathways likely has important therapeutic implications for many chronic conditions. ${ }^{15}$

While many studies have evaluated "basal" or "resting" levels of cytokines in chronic musculoskeletal pain conditions, some investigators have measured changes in IL-6 or other cytokines in response to a standardized stressor, or stimulus. ${ }^{16-19}$ Studies looking at aging have demonstrated decreased production of proinflammatory cytokines after exposure to lipopolysaccharide, which may reflect impaired host defense against infections in the elderly. ${ }^{20}$ Several reports have also indicated that IL-6 levels increase in response to standardized pain-provoking procedures (such as administration of noxious mechanical stimuli), ${ }^{21}$ though overall, the literature linking acute pain and inflammatory responses has been rather inconsistent. ${ }^{22-24}$ We have used such procedures (eg, application of standardized painful stimulation and measurement of changes in circulating cytokine levels) as a way to quantify individual variability in cytokine reactivity to study the effects of psychosocial variables such as painrelated catastrophizing on physiological pain responses. ${ }^{25}$ A number of psychoneuroimmunology studies have linked psychosocial states with inflammatory processes, and catastrophizing is one of the psychosocial factors that have been most extensively studied as a contributor to pain-related inflammation. ${ }^{25-27}$ Both pain catastrophizing and anxiety are characterized by attention to threat, overemphasis of the probability of a catastrophic outcome, and rumination about the worst possible consequences. ${ }^{28-30}$ Despite this overlap with anxiety, pain catastrophizing seems to play a unique role in pain experience. For instance, Pinto et al have demonstrated that pain catastrophizing fully mediated (ie, statistically accounted for) the association between presurgical anxiety and postsurgical pain intensity. ${ }^{31}$ In our previous studies, we demonstrated that pain catastrophizing, measured immediately after the pain procedures, was related to IL-6 reactivity even after controlling for other negative affective states. ${ }^{25}$

Catastrophizing consists of a set of negative cognitive and emotional experiences involving rumination about pain, feelings of helplessness when in pain, and pain-magnification of the threat value of pain. ${ }^{32-36}$ There have been some previous studies exploring the subscales of pain catastrophizing as separate constructs. Specifically, there has been an interest in pain-magnification, defined as a heightened perception of the threat represented by pain symptoms. ${ }^{37}$ Recent studies have demonstrated that only pain pain-magnification and state anxiety emerged as significant predictors of pain intensity. ${ }^{38}$ In addition, both helplessness and pain-magnification were related to mental health-related QOL and depression, whereas only pain pain-magnification has been shown to be associated with physical health-related QOL. ${ }^{39}$

Individuals who score high on measures of catastrophizing tend to exhibit increased hyperalgesia, higher ratings of chronic pain intensity, and elevated levels of physical disability. ${ }^{25}$ Catastrophizing appears to be linked with some of the neurobiological mechanisms underlying pain perception, including inflammation. ${ }^{25}$ For example, recent studies find a positive correlation between catastrophizing and elevated indices of inflammation in rheumatoid arthritis. ${ }^{25,40-42}$ Longitudinal RA studies have shown that high levels of catastrophizing prospectively predicted worsening erythrocyte sedimentation rates. ${ }^{43}$ Of note, a study in healthy adults revealed that higher levels of catastrophizing predicted greater IL-6 reactivity to standardized noxious stimulation in a laboratory setting, ${ }^{25}$ and O'Donovan et al directly compared anxious and non-anxious individuals and demonstrated that clinically anxious participants exhibited significantly higher levels of IL- $6 .{ }^{44}$ Catastrophizing also interacts with other negative affective processes: in a recent study, Sturgeon and Zautra $^{45}$ demonstrated that pain catastrophizing accounted for a significant proportion of the relationship between daily pain intensity, negative and positive affect, as well as depressive symptoms. Collectively, however, little research has explored the interrelationships between catastrophizing and anxiety in the context of pain and inflammation.

The primary aim of the present study was to examine, in a controlled manner, the influence of negative cognitive/ emotional states such as pain catastrophizing and anxiety on the magnitude and time course of IL-6 responses among 
patients with chronic pain following a session of laboratoryinduced pain. In order to isolate the specific effects of pain, we included a nonpainful laboratory testing session as well as a comparison group of healthy controls. The IL-6 reactivity of FM and OA patients during a painful Quantitative Sensory Testing (QST) session was compared with IL-6 responses obtained following a nonpainful laboratory session, and with those of a demographically comparable group of healthy, pain-free controls.

\section{Patients and methods Patients}

A total of 48 participants were enrolled in the study, with 12 healthy volunteers and 36 chronic pain patients (FM and $\mathrm{OA})$. The groups were selected to be comparable in age, ethnicity, and sex, as these demographic factors correlate with both pain experience and immune system functioning. ${ }^{46,47}$ Most of the participants were women, since there is a strong female predominance in epidemiological studies of arthritis and FM. Catastrophizing, which often differs systematically across groups, was not used as a matching variable and was permitted to vary freely. The involvement of human subjects in this study was reviewed, approved, and monitored by the Institutional Review Board at Brigham and Women's hospital, Boston, MA, USA, and all participants provided written informed consent. Participants were screened using the following inclusion criteria for FM and OA patients: 1) 40-70 years old, and must meet the clinical criteria for a chronic pain musculoskeletal disorder (eg, OA, FM); 2) typical pain ratings $\geq 3$ on the Brief Pain Inventory (BPI) Pain Intensity subscale; and 3) facility with the English language that is adequate to complete study procedures. The exclusion criteria for the patients were as follows: 1) delirium, dementia, psychosis, or other cognitive impairment preventing completion of study procedures; 2 ) history of myocardial infarction or other serious cardiovascular condition; 3) current peripheral neuropathy; 4) currently pregnant; 5) history of Raynaud's syndrome; 6) active vasculitis or severe peripheral vascular disease; 7) current use of tumor necrosis factor-alpha antagonists or steroids; 8) current infection; 9) history of autoimmune disorder such as systemic lupus erythematosus; 10) use of strong opioids; and 11) recent history of substance abuse or dependence.

The inclusion criteria for healthy controls were the following: 1) 40-70 years old, and 2) facility with the English language that is adequate to complete study procedures. The exclusion criteria for the controls were identical to those for the patients, with the following two additions: 1) significant current or recent pain complaints, and 2) a history of any painful musculoskeletal disorder (eg, OA, FM).

\section{Procedures}

All participants underwent two testing sessions: one with standard (painful) QST procedures (described below) and the other with equivalent procedures performed in a nonpainful manner. The order of testing sessions (painful and nonpainful) was randomized. Visits were scheduled in the morning, starting at 8:00 am. All participants were instructed not to eat and drink for at least 2 hours beforehand, not to perform strenuous exercise that morning, and not to use antiinflammatory medications prior to the testing session. Blood samples were collected twice at baseline during a 20-minute rest period (prior to QST), after the QST, and at 30 minutes, 1 hour, 90 minutes, and 2 hours post-QST. The total duration of the painful QST was 30 minutes. Participants were seated comfortably in a reclining chair and an indwelling catheter was inserted in the left forearm. Blood samples were collected in $10 \mathrm{~mL}$ tubes and transported to the laboratory, where they were centrifuged, aliquoted, and stored in a $-70^{\circ} \mathrm{C}$ freezer. A standard high-sensitivity enzyme-linked immunosorbent assay (R\&D Systems, Minneapolis, MN, USA) was used to assess serum levels of IL-6 (lower limit of detection: $0.16 \mathrm{pg}$ / $\mathrm{mL}$; sensitivity: $0.04 \mathrm{pg} / \mathrm{mL}$; intra-assay coefficient of variation: $<5 \%$ ), a proinflammatory cytokine which is associated with peripheral and central sensitization. ${ }^{48,49}$

At the painful QST visit, the participants underwent noxious mechanical and cold stimulation, similar to our previous studies. ${ }^{25,50,51}$ First, pressure pain thresholds (PPTh) were determined bilaterally at the trapezius muscle, the metacarpophalangeal joint of the thumb, and the patella using a Somedic digital pressure algometer. This PPTh procedure lasted $\sim 15$ minutes. Next, we assessed tonic deep-tissue pressure pain by inflating a blood pressure cuff around the lower leg, over the gastrocnemius muscle, to an individually tailored moderately painful level. This cuff pain testing procedure lasted $\sim 5$ minutes. Finally, the participants underwent a series of four cold pressor tasks involving immersion of the hand in a circulating $4^{\circ} \mathrm{C}$ water bath. Each cold water immersion lasted $\sim 45$ seconds, with a 2-minute rest period in between each immersion. This procedure lasted $\sim 10$ minutes.

At the nonpainful QST visit, the same procedures were performed for the same durations, but without inducing pain. This was done by assessing the thresholds for mild, nonpainful pressure using the algometer, by applying cuff stimulation that was tailored to an intensity that produced sensations of mild, nonpainful pressure $(30 \mathrm{mmHg})$, and by 
having participants undergo a series of hand immersions in a water bath maintained at $26^{\circ} \mathrm{C}$.

Participants completed standard questionnaires such as the BPI, ${ }^{52}$ the Pain Catastrophizing Scale (PCS), ${ }^{53}$ and the Beck Depression Inventory. ${ }^{54}$ In addition, participants reported on their current clinical pain and current anxiety (state anxiety) at multiple time points throughout the session, as in previous QST studies by our group. ${ }^{55,56}$

\section{Data analysis}

All analyses were conducted using Statistical Package for the Social Sciences (SPSS) version 21.0 (IBM Corp., Armonk, NY, USA). Descriptive data for continuous variables are presented as means and standard deviations (SDs), and data for categorical variables are presented as percentages. All analyses were conducted using multilevel modeling (MLM) and the MIXED command in SPSS-IBM. MLM is well-suited to handle the hierarchical nested data structure of the proposed study, in which repeated daily assessments (Level 1 units) were nested within participants. MLM is also well-suited to handle the unequal number of data points across participants due to random missing data, ${ }^{57,58}$ which is typical of longitudinal study designs.

Data cleaning included correction of out-of-range values and checking for potential outliers. The multivariate outlier analysis was conducted using the Mahalanobis distance procedure, separately for the noxious and non-noxious conditions. For the noxious condition, analyses revealed one multivariate outlier case for the anxiety-IL6 association and two outlier cases for the pain-magnification-IL6 association. Sensitivity analyses were subsequently conducted to determine whether the presence/absence of these outlier cases influenced the main study findings reported in the initial version of the manuscript. Sensitivity analyses indicated that the main multilevel model (ie, time * pain-magnification on IL-6 responses) among pain patients remained significant $(p<0.05)$ even when removing these outlier cases. Analyses also indicated that this model was nonsignificant among healthy controls $(p>0.05)$.

We also examined the amount of missing data for each of the main independent variables (pain-magnification, anxiety) and for the study outcome (IL-6). Across the noxious and non-noxious conditions, there was no missing data $(0 \%)$ for the IL-6 variable and no missing data for the pain-magnification variable $(0 \%)$. There were, however, missing data for the anxiety ratings collected during the noxious (16.1\%) and non-noxious (15.1\%) conditions.

We first conducted preliminary analyses examining the potential confounding influence of participants' demographic (ie, age, sex, ethnicity) and medication use (ie, opioids, antidepressants, anxiolytics/sedatives, anticonvulsants, muscle relaxants) characteristics on IL-6 responses. Consistent with recommendations, ${ }^{59,60}$ variables that were significantly associated with IL-6 were retained as covariates in the primary multilevel models described below. For example, emotional distress (eg, depression) seems to affect IL-6 levels and is often used as a covariate. ${ }^{61}$ More specifically, Craner et al ${ }^{39}$ have previously shown that pain-magnification, one of the main domains of catastrophizing, was significantly related to physical and mental health-related QOL and depressed mood in patients with chronic pain. In addition, previous studies have shown a strong association between pain-magnification, anxiety, and depression in chronic pain samples; ${ }^{62}$ indeed, one study found that pain-magnification was the only domain of catastrophizing that was uniquely associated with negative emotions such as anxiety. ${ }^{63}$

In order to select the covariance structure for multilevel models, we compared different types of covariance structures (ie, first-order autoregressive [AR1], compound symmetry, Toeplitz) by examining model fit information. Comparison of model fit was based on the Akaike information criterion and the Schwarz's Bayesian information criterion, as recommended. ${ }^{64}$ Model fit comparisons were made separately for models examining IL-6 responses in the noxious and nonnoxious conditions, as well as separately for pain patients and healthy controls. Across all models, the best model fit was achieved by using the AR1 structure. In addition to providing the best model fit, the AR1 structure permits accounting for the autocorrelations between repeated outcome assessments (ie, IL-6) during testing sessions. For all these reasons, the AR1 structure was selected for our multilevel models.

All multilevel models were built using IL-6 responses as the dependent (ie, outcome) variable. "Time" was first included as a Level 1 independent variable, and Level 1 anxiety and Level 2 catastrophizing (the pain-magnification subscale of the PCS) scores were then simultaneously added to the model, which permitted examination of the effects of catastrophizing (pain-magnification) on IL-6 responses, controlling for anxiety. In order to examine whether catastrophizing influenced the time course of IL-6 responses, a two-way (time * Level 2 catastrophizing/pain-magnification) interaction term was then specified and included in the model, controlling for anxiety.

As recommended, all Level 1 scores were centered within participants and Level 2 scores were centered at the grand mean. ${ }^{65,66}$ Model building followed a sequential procedure, ${ }^{67-69}$ which first involved specifying a random intercept and fixed effects for independent variables. When 
significant fixed effects emerged, slopes were then treated as random effects, and model fit was re-evaluated using the likelihood ratio test. Random parameters were dropped if they resulted in a significantly worse model fit. ${ }^{70-72}$ All models used maximum-likelihood estimation and included an AR1 variance covariance matrix in order to account for autocorrelations between repeated assessments.

In addition, mean group differences between chronic pain patients and healthy controls were compared using one-way analysis of variance.

\section{Results \\ Descriptive statistics}

Descriptive statistics for study measures are presented in Tables 1 and 2, separately for chronic pain patients and healthy controls. The average age of patients was 51.9 years $(\mathrm{SD}=9.1)$ and the majority were women. Scores on pain (ie, BPI) and psychological (ie, PCS) measures are comparable

Table I Sociodemographic and clinical variables

\begin{tabular}{lll}
\hline & $\begin{array}{l}\text { Healthy controls } \\
(\mathbf{n = 1 2})\end{array}$ & $\begin{array}{l}\text { Chronic pain } \\
\text { participants (n=36) }\end{array}$ \\
\hline Age (years), mean $\pm \mathrm{SD}$ & $51.5 \pm 13.2$ & $51.7 \pm 7.6$ \\
Female & $66.7 \%$ & $86.1 \%$ \\
Married & $25 \%$ & $33.3 \%$ \\
Caucasian & $63.6 \%$ & $58.3 \%$ \\
Employed & $45.5 \%$ & $11.1 \%$ \\
College degree & $54.5 \%$ & $36.1 \%$ \\
Body mass index $\left(\mathrm{kg} / \mathrm{m}^{2}\right)$, & $25.9 \pm 3.3$ & $28.6 \pm 7.7$ \\
mean \pm SD & & \\
Antidepressants & $8.3 \%$ & $44.4 \%$ \\
Weak opioids & $0 \%$ & $16.7 \%$ \\
Anxiolytics/sedatives & $0 \%$ & $33.3 \%$ \\
Anticonvulsants & $0 \%$ & $22.2 \%$ \\
Muscle relaxants & $0 \%$ & $13.9 \%$ \\
\hline
\end{tabular}

to those that have been observed in previous studies among patients with chronic pain. ${ }^{73-75}$

Before conducting primary analyses, the potential confounding influence of patient demographics (ie, age, sex, ethnicity) and medication use on IL-6 responses was examined. Given that none of these variables were significantly associated with IL-6 (all $p$ values $>0.05$ ), they were not included as covariates in the multilevel models reported below.

We also compared the groups on responses to QST in the painful session (eg, PPTh, cold pressor tolerance) as well as psychosocial measures (PCS, BPI, Beck Depression Inventory, anxiety, and clinical pain) at baseline. Chronic pain patients demonstrated significant differences from controls on most measures (Table 2).

\section{Time course of IL-6 responses during the painful session}

A multilevel model was built to examine the time course of IL-6 responses during the noxious condition among pain patients (Table 3; Figure 1). In this model, "time" was used as a Level 1 independent variable and IL-6 as the dependent variable (ie, outcome). Results revealed a significant main effect of time $(\mathrm{B}=2.35$, standard error $[\mathrm{SE}]=0.64, p<0.001)$, indicating that IL-6 responses increased across assessment time points. Level 2 catastrophizing scores were then added to the model; the main effect of catastrophizing was not significant $(B=0.12$, $\mathrm{SE}=0.26$ ). Results revealed a significant two-way (time * catastrophizing) interaction effect $(\mathrm{B}=0.036, \mathrm{SE}=0.016$, $p<0.05)$, revealing the influence of pain-magnification on the time course of IL-6 responses over time. When controlling for anxiety, results indicated that the main multilevel model (ie, time * pain-magnification on IL-6 responses) among patients remained significant $(\mathrm{B}=0.034, \mathrm{SE}=0.017, p<0.05)$.

Table 2 Comparison of patient and control groups (data presented as mean \pm SD) at baseline

\begin{tabular}{|c|c|c|c|}
\hline & Healthy controls & Patients & $p$-value \\
\hline \multicolumn{4}{|l|}{ Responses to noxious stimuli } \\
\hline PPTh trapezius (kPa) & $404.5 \pm 173.0$ & $245.5 \pm 116.3$ & $<0.001$ \\
\hline Cuff pressure $(\mathrm{mmHg})$ & $216.9 \pm 79.0$ & $|32.3| \pm 6 \mid .7$ & $<0.001$ \\
\hline Cold pressor tolerance (seconds) & $86.82 \pm 64.5$ & $65.1 \pm 50.4$ & ns \\
\hline Cold pain rating $(0-100)$ & $69.42 \pm 25.2$ & $80.45 \pm 23.1$ & ns \\
\hline \multicolumn{4}{|l|}{ Psychosocial measures } \\
\hline BPI (severity) & 0 & $4.3 \pm 2.0$ & $<0.001$ \\
\hline BPI (interference) & 0 & $5.0 \pm 2.3$ & $<0.001$ \\
\hline BDI & $2.3 \pm 3.2$ & $12.7 \pm 8.9$ & $<0.001$ \\
\hline PCS (total score) & $5.8 \pm 7.9$ & $18.8 \pm 12.1$ & $<0.001$ \\
\hline Clinical anxiety $(0-10)$ & I I. $2 \pm 4.0$ & $16.4 \pm 6.0$ & $<0.01$ \\
\hline Clinical pain (0-10) & $0.2 \pm 0.6$ & $3.59 \pm 2.4$ & $<0.001$ \\
\hline
\end{tabular}

Abbreviations: PPTh, pain pressure thresholds; BDI, Beck Depression Inventory; BPI, Brief Pain Inventory; ns, nonsignificant; PCS, Pain Catastrophizing Scale. 
Table 3 Multilevel model examining the influence of state anxiety and trait catastrophizing (pain-magnification) on the time course of IL-6 responses during the painful session among participants with chronic pain

\begin{tabular}{|c|c|c|c|c|}
\hline Fixed effects & $\boldsymbol{\beta}$ & SE & $\boldsymbol{t}$ & $p$-value \\
\hline \multicolumn{5}{|l|}{ Step I } \\
\hline Intercept & 2.35 & 0.644 & 3.65 & $\leq 0.001$ \\
\hline Time & 0.263 & 0.039 & 6.70 & $\leq 0.001$ \\
\hline Pain-magnification (PCS) & 0.12 & 0.27 & 0.47 & ns \\
\hline Time $\times$ pain-magnification $(\mathrm{PCS})$ & 0.036 & 0.013 & 2.24 & $<0.05$ \\
\hline \multicolumn{5}{|l|}{ Step 2} \\
\hline Intercept & 3.23 & 0.88 & 3.67 & $\leq 0.001$ \\
\hline Time & 0.26 & 0.0439 & 6.70 & $\leq 0.001$ \\
\hline Anxiety & -0.05 & 0.041 & -1.42 & ns \\
\hline Pain-magnification (PCS) & 0.22 & 0.27 & 0.81 & ns \\
\hline Time $\times$ pain-magnification $(\mathrm{PCS})$ & 0.036 & 0.016 & 2.25 & $<0.05$ \\
\hline
\end{tabular}

Notes: Time and anxiety are Level I variables centered within participants. Catastrophizing is a Level 2 variable centered at the grand mean. $\beta$, unstandardized regression coefficient.

Abbreviations: IL, interleukin; ns, nonsignificant; PCS, Pain Catastrophizing Scale; SE, standard error.

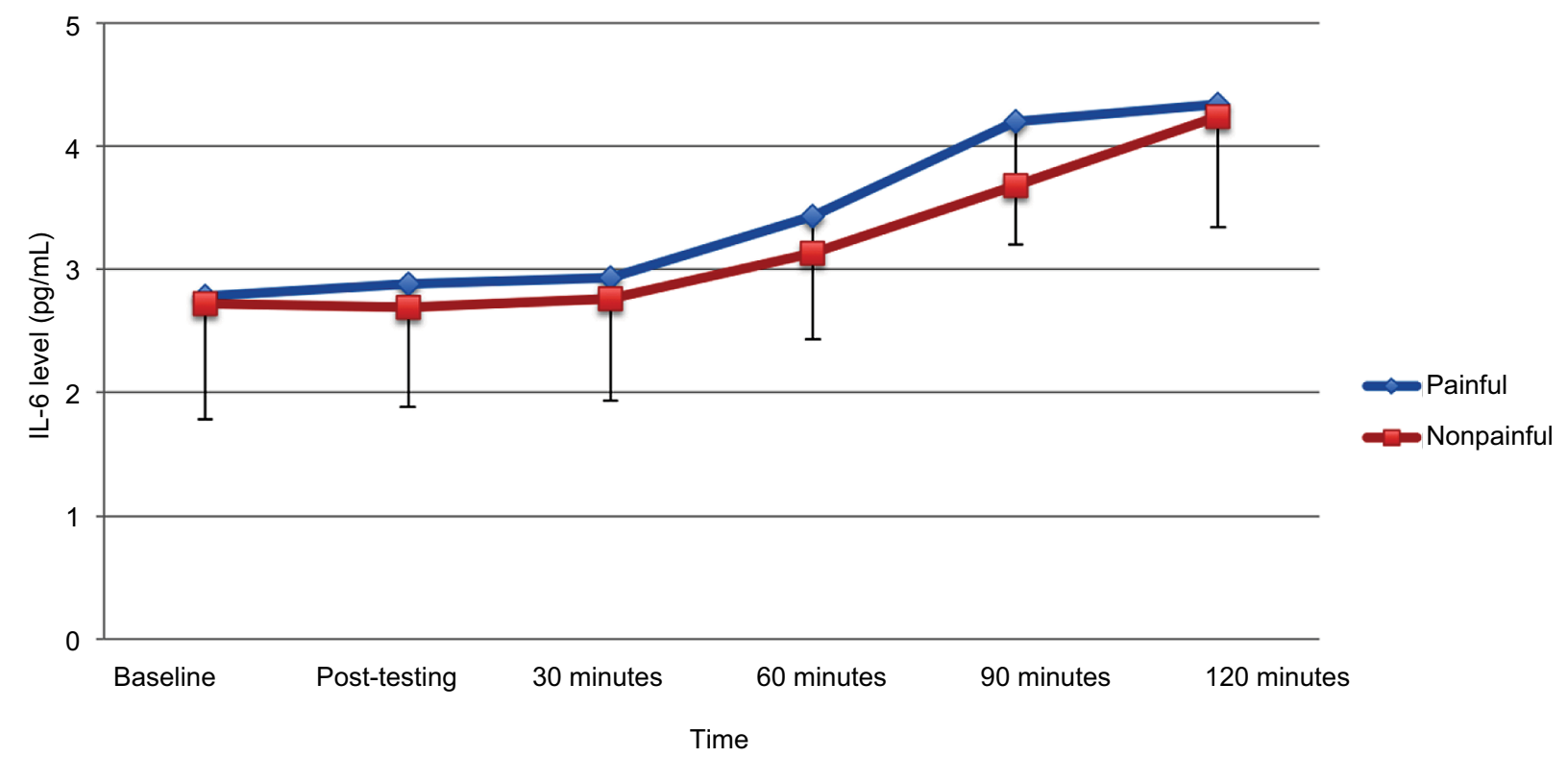

Figure I IL-6 levels in chronic pain patients (FM and OA). Data points are group means; error bars represent standard deviation. Abbreviations: FM, fibromyalgia; IL-6, interleukin-6; OA, osteoarthritis.

A subsequent multilevel model was then built following the same model-building approach as described above, but among healthy controls (Figure 2). Results revealed a significant main effect of time $(\mathrm{B}=0.22, \mathrm{SE}=0.072, p<0.01)$, indicating that IL- 6 responses increased across assessment time points. Results also revealed no significant main effect of pain-magnification $(\mathrm{B}=0.1 .86, \mathrm{SE}=2.11, p<0.05)$. Time was not involved in any significant two-way interaction effect (both $p$ values $>0.05$ ), indicating that the time course of IL-6 responses was not significantly influenced by anxiety or catastrophizing.

Time course of IL- 6 responses during the nonpainful session

A multilevel model was built to examine the time course of IL-6 responses during the non-noxious condition among pain patients (Table 4; Figure 2). In this model, "time" was again used as a Level 1 independent variable and IL-6 as the 


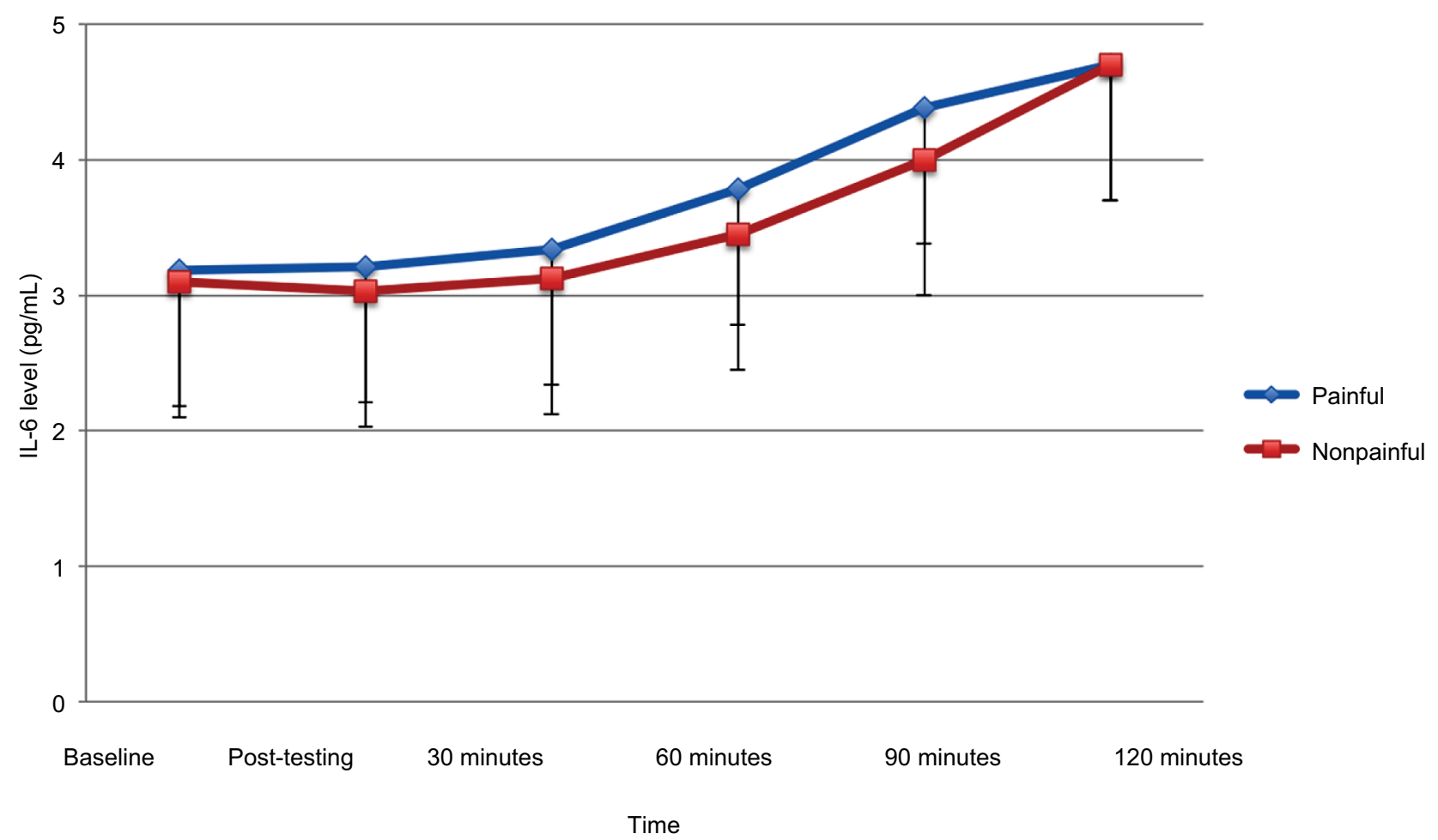

Figure 2 Interleukin-6 (IL-6) levels in healthy controls. Data points are group means; error bars represent standard deviation.

Table 4 Multilevel model examining the influence of state anxiety and trait catastrophizing on the time course of IL-6 responses during the nonpainful session among participants with chronic pain

\begin{tabular}{lllll}
\hline Fixed effects & $\boldsymbol{\beta}$ & $\mathbf{S E}$ & $\boldsymbol{t}$ & $\boldsymbol{p}$-value \\
\hline Step I & & & & \\
Intercept & 2.50 & 0.635 & 3.94 & $<0.001$ \\
Time & 0.243 & $0.05 \mathrm{I}$ & 4.76 & $\mathrm{n}$ \\
Pain-magnification (PCS) & 0.16 & 0.25 & 0.66 & $\mathrm{~ns}$ \\
Time $\times$ pain-magnification (PCS) & -0.028 & 0.020 & -1.40 & \\
Step 2 & & & & \\
Intercept & 2.43 & 0.62 & 3.89 & $<.001$ \\
Time & 0.25 & 0.052 & 4.76 & $\mathrm{~ns}$ \\
Anxiety & 0.0002 & 0.005 & 0.038 & $\mathrm{~ns}$ \\
Pain-magnification (PCS) & 0.178 & 0.021 & 0.709 & $\mathrm{~ns}$ \\
Time $\times$ pain-magnification (PCS) & -0.032 & 0.021 & -1.54 & \\
\hline
\end{tabular}

Notes: Time and anxiety are Level I variables centered within participants. Catastrophizing is a Level 2 variable centered at the grand mean. $\beta$, unstandardized regression coefficient.

Abbreviations: IL, interleukin; ns, nonsignificant; PCS, Pain Catastrophizing Scale; SE, standard error.

dependent variable (ie, outcome). Results revealed a significant main effect of time $(\mathrm{B}=0.244, \mathrm{SE}=0.050, p<0.001)$, indicating that IL-6 responses increased across assessment time points. Level 2 catastrophizing scores were then added to the model, but did not significantly contribute to the prediction of IL-6 (both $p$ values $>0.05$ ). When controlling for anxiety, results indicated that the main multilevel model (ie, time * pain-magnification on IL-6 responses) among patients remained nonsignificant $(p>0.05)$. Time was not involved in any significant two-way interaction effect (both $p$ values $>0.05$ ), indicating that the time course of IL- 6 responses was not significantly influenced by anxiety or catastrophizing.

Analyses of variance examining group differences in IL-6 responses revealed no differences between chronic 
pain patients and healthy controls in IL-6 levels for either the painful or nonpainful testing sessions (both $p$ values $>0.05$ ). Moreover, no significant differences in IL-6 levels emerged when comparing the painful and nonpainful sessions ( $p$ values $>0.05$ ). We also explored the relationship between patients' reported clinical pain intensity and the QST-based indices of pain sensitivity. Pearson's correlation analysis revealed significant correlations between the clinical pain intensity at the painful session and the QST parameters that showed elevated pain sensitivity for the patient group (ie, trapezius pain threshold, cuff pressure); $r=0.37$ for trapezius pain threshold and $r=0.53$ for cuff pressure ( $p$ values $<0.05$ ), indicating that more intense clinical pain was associated with enhanced pain sensitivity within the patient group.

\section{Discussion}

In this study, we examined the psychosocial influences on IL-6 reactivity among chronic pain patients and controls undergoing standardized painful and nonpainful stimulation. Results of multilevel growth curve analyses indicated that IL-6 levels showed a mild increase over the course of the testing procedure for both groups in both sessions (ie, across assessment time points). These findings are congruent with previous reports on increased IL-6 levels following QST in healthy adults ${ }^{25}$ as well as in patients with arthritis. ${ }^{51,76}$ Unlike previous work, the present study included both a nonpainful and a painful session, providing a controlled framework for exploring potential unique psychosocial contributors to the impact of painful stimulation on cytokine responses. ${ }^{76}$ The observed increase in IL-6 is concordant with previous studies, ${ }^{25,51,77,78}$ but the similarity of the increase in the nonpainful testing session suggests that such IL-6 reactivity is not pain specific. Both patients and healty controls demonstrated an increase in IL-6 levels during testing, which may suggest that the observed effects are due to a general stress response, ${ }^{79}$ circadian rhythmicity, or possibly an effect of local inflammatory responses to repeated blood draws from a catheter. ${ }^{80}$ The observed increases in plasma concentrations of IL-6 were small relative to the changes observed after manipulations such as endotoxin (eg, lipopolysaccharide) injection (acute inflammatory response to endotoxin in mice and humans), ${ }^{81}$ so the clinical implications are uncertain. However, even fairly slight variation in the circulating levels of proinflamamtory cytokine concentrations may have implications for the experience of musculoskeletal pain. ${ }^{82}$

The main finding that emerged from our study was that the pain-magnification component of catastrophizing moderated IL-6 increases over time in patients with chronic pain.
Multilevel analyses indicated that higher pain-magnification scores were associated with heightened IL-6 responsiveness to painful stimulation. The influence of catastrophizing on IL-6 responses is consistent with the findings of several studies that have also documented positive associations of IL-6 with indices of emotional distress. ${ }^{83}$ The findings were unique to the chronic pain patients and to the painful testing session, highlighting the potential specificity of this effect to pain-related stress responses. ${ }^{33,36}$ Overall, our findings are in broad agreement with previous results suggesting that negative cognitive/emotional factors such as catastrophizing are related to indices of inflammatory disease activity or proinflammatory immunologic parameters in chronic pain patients. ${ }^{84-86}$ The present study extends this general effect, indicating that this inflammatory stress response may be selective to patients with persistent pain who experience a high degree of catastrophizing cognitions (specifically those involving pain-magnification and amplification of the threat value of pain) in response to a pain-related stressor. It is interesting to consider that other biopsychosocial forces may be involved in this association; for example, recent reviews of the effects of cytokines on human functioning suggest links with social processes ${ }^{87}$ and catastrophizing has also been implicated in shaping social interactions. ${ }^{88}$ The present study was not performed to investigate this hypothesis, but future studies may benefit from considering whether cytokines represent one of the pathways by which catastrophizing and patients' social environments are reciprocally interrelated.

Our findings indicated that there are no differences between chronic pain patients and healthy controls in release of proinflammatory cytokines, confirming the results from previous studies. ${ }^{89}$ However, catastrophizing seems to be related to proinflammatory pain-stress response in the chronic pain patient group and in the painful session only. Collectively, the findings of this study have implications for the psychological mechanisms underlying stress responses in the context of pain. Some researchers ${ }^{90}$ have suggested that indices of low mood (ie, depression) could be used as direct proxy measures of chronic inflammation, and numerous investigators observed that stress ${ }^{91,92}$ and depression ${ }^{93,94}$ are associated with increased levels of circulating proinflammatory cytokines, particularly IL-6. However, there has been relatively less investigation of the relationships between anxiety, catastrophizing, and inflammatory activity in chronic pain. In addition, the recent literature on state anxiety and inflammation has shown mixed patterns of results. ${ }^{91,95}$ These largely cross-sectional studies fail to shed light on the temporal aspects of the interactions between psychological states 
and inflammation; while it is clear that stressful events can trigger an inflammatory response, proinflammatory cytokines can induce symptomatology consistent with states of depression and anxiety. ${ }^{96,97}$ Our study cannot untangle the complex dynamics of the interplay between emotions and the immune system, but it does suggest that there may be particular psychological characteristics (eg, catastrophizing and pain-magnification-related cognitions) that impact proinflammatory stress reactivity in chronic pain patients.

The limitations of this study should be recognized when interpreting these results. First, the sample size was relatively small, especially in the control group, preventing us from drawing clear conclusions regarding differences in IL-6 levels or reactivity across groups. The small size of the control group also made it difficult to precisely match the samples on factors such as sex. Second, the chronic pain population included both OA and FM patients, which likely added to the heterogeneity of our sample. In addition, we were not able to fully standardize variables such as wake time on the day of testing, which would likely have required an inpatient study. Although we standardized the time of day that testing and sample collection occurred, there are a large number of circadian factors that can influence cytokine levels. ${ }^{98}$ Third, we did not obtain genotypic data on participants, which may have helped to predict individual variability in cytokine reactivity, as such responses are known to be influenced by a variety of genes including those involved in the cholinergic system. ${ }^{99}$ Finally, we studied only IL-6, a quintessential proinflammatory cytokine, and the increases demonstrated were relatively small; in future studies, we plan to consider assaying larger panels of both pro- and anti-inflammatory cytokines. Despite these limitations, the current findings are encouraging and we hope that they will stimulate further investigation into the links between psychological and inflammatory processes in the context of chronic pain.

\section{Acknowledgment}

The authors wish to acknowledge Dr Gayle Page for her guidance on the proinflammatory cytokine data acquisition and analysis.

\section{Disclosure}

The authors report no conflicts of interest in this work.

\section{References}

1. March L, Smith EU, Hoy DG, et al. Burden of disability due to musculoskeletal (MSK) disorders. Best Pract Res Clin Rheumatol. 2014;28(3):353-366.
2. Clauw DJ. Fibromyalgia: more than just a musculoskeletal disease. $A m$ Fam Physician. 1995;52(3):843-851.

3. Clauw DJ. Fibromyalgia: update on mechanisms and management. J Clin Rheumatol. 2007;13(2):102-109.

4. Skaer TL. Fibromyalgia: disease synopsis, medication cost effectiveness and economic burden. Pharmacoeconomics. 2014;32(5): 457-466.

5. Cross M, Smith E, Hoy D, et al. The global burden of hip and knee osteoarthritis: estimates from the global burden of disease 2010 study. Ann Rheum Dis. 2014;73(7):1323-1330.

6. Rodriguez-Pinto I, Agmon-Levin N, Howard A, Shoenfeld Y. Fibromyalgia and cytokines. Immunol Lett. 2014;161(2):200-203.

7. Greene MA, Loeser RF. Aging-related inflammation in osteoarthritis. Osteoarthritis Cartilage. 2015;23(11):1966-1971.

8. Agmon-Levin N, Zafrir Y, Kivity S, Balofsky A, Amital H, Shoenfeld Y. Chronic fatigue syndrome and fibromyalgia following immunization with the hepatitis B vaccine: another angle of the 'autoimmune (autoinflammatory) syndrome induced by adjuvants' (ASIA). Immunol Res. 2014;60(2-3):376-383.

9. Imamura M, Targino RA, Hsing WT, et al. Concentration of cytokines in patients with osteoarthritis of the knee and fibromyalgia. Clin Interv Aging. 2014;9:939-944.

10. van West $D$, Maes M. Neuroendocrine and immune aspects of fibromyalgia. BioDrugs. 2001;15(8):521-531.

11. Uceyler N, Hauser W, Sommer C. Systematic review with meta-analysis: cytokines in fibromyalgia syndrome. BMC Musculoskelet Disord. 2011;12:245.

12. Doss F, Menard J, Hauschild M, et al. Elevated IL-6 levels in the synovial fluid of osteoarthritis patients stem from plasma cells. Scand J Rheumatol. 2007;36(2):136-139.

13. Imamura M, Ezquerro F, Marcon Alfieri F, et al. Serum levels of proinflammatory cytokines in painful knee osteoarthritis and sensitization. Int J Inflam. 2015;2015:329792.

14. Rosas-Ballina M, Tracey KJ. Cholinergic control of inflammation. J Intern Med. 2009;265(6):663-679.

15. Olshansky B. Vagus nerve modulation of inflammation: Cardiovascular implications. Trends Cardiovasc Med. 2016;26(1):1-11.

16. Elenkov IJ, Iezzoni DG, Daly A, Harris AG, Chrousos GP. Cytokine dysregulation, inflammation and well-being. Neuroimmunomodulation. 2005;12(5):255-269.

17. Woda A, Picard P, Dutheil F. Dysfunctional stress responses in chronic pain. Psychoneuroendocrinology. 2016;71:127-135.

18. Tian R, Hou G, Li D, Yuan TF. A possible change process of inflammatory cytokines in the prolonged chronic stress and its ultimate implications for health. ScientificWorldJournal. 2014;2014:780616.

19. Dantzer R, Kelley KW. Twenty years of research on cytokine-induced sickness behavior. Brain Behav Immun. 2007;21(2):153-160.

20. Bruunsgaard H, Pedersen AN, Schroll M, Skinhoj P, Pedersen BK. Impaired production of proinflammatory cytokines in response to lipopolysaccharide (LPS) stimulation in elderly humans. Clin Exp Immunol. 1999;118(2):235-241.

21. Geiss A, Varadi E, Steinbach K, Bauer HW, Anton F. Psychoneuroimmunological correlates of persisting sciatic pain in patients who underwent discectomy. Neurosci Lett. 1997;237(2-3):65-68.

22. Bazzichi L, Dini M, Rossi A, et al. Muscle modifications in fibromyalgic patients revealed by surface electromyography (SEMG) analysis. BMC Musculoskelet Disord. 2009;10:36.

23. Gur A, Karakoc M, Nas K, et al. Cytokines and depression in cases with fibromyalgia. J Rheumatol. 2002;29(2):358-361.

24. Wang H, Moser M, Schiltenwolf M, Buchner M. Circulating cytokine levels compared to pain in patients with fibromyalgia - a prospective longitudinal study over 6 months. $J$ Rheumatol. 2008;35(7): $1366-1370$.

25. Edwards RR, Kronfli T, Haythornthwaite JA, Smith MT, McGuire L, Page GG. Association of catastrophizing with interleukin-6 responses to acute pain. Pain. 2008;140(1):135-144. 
26. Sloan TJ, Gupta R, Zhang W, Walsh DA. Beliefs about the causes and consequences of pain in patients with chronic inflammatory or noninflammatory low back pain and in pain-free individuals. Spine (Phila Pa 1976). 2008;33(9):966-972.

27. Parker JC, Smarr KL, Angelone EO, et al. Psychological factors, immunologic activation, and disease activity in rheumatoid arthritis. Arthritis Care Res. 1992;5(4):196-201.

28. Grant DM, Beck JG. What predicts the trajectory of rumination?: a prospective evaluation. J Anxiety Disord. 2010;24(5):480-486.

29. Sullivan MJ, Neish NR. Catastrophizing, anxiety and pain during dental hygiene treatment. Community Dent Oral Epidemiol. 1998;26(5):344-349.

30. Turner JA, Aaron LA. Pain-related catastrophizing: what is it? Clin J Pain. 2001;17(1):65-71.

31. Pinto PR, McIntyre T, Almeida A, Araujo-Soares V. The mediating role of pain catastrophizing in the relationship between presurgical anxiety and acute postsurgical pain after hysterectomy. Pain. 2012;153(1):218-226.

32. Campbell CM, Kronfli T, Buenaver LF, et al. Situational versus dispositional measurement of catastrophizing: associations with pain responses in multiple samples. J Pain. 2010;11(5):443-453. e442.

33. Edwards RR, Bingham CO 3rd, Bathon J, Haythornthwaite JA. Catastrophizing and pain in arthritis, fibromyalgia, and other rheumatic diseases. Arthritis Rheum. 2006;55(2):325-332.

34. Leung L. Pain catastrophizing: an updated review. Indian J Psychol Med. 2012;34(3):204-217.

35. Sullivan MJ, Martel MO, Tripp D, Savard A, Crombez G. The relation between catastrophizing and the communication of pain experience. Pain. 2006;122(3):282-288.

36. Edwards RR, Cahalan C, Mensing G, Smith M, Haythornthwaite JA. Pain, catastrophizing, and depression in the rheumatic diseases. Nat Rev Rheumatol. 2011;7(4):216-224.

37. Sullivan MJ, Thorn B, Haythornthwaite JA, et al. Theoretical perspectives on the relation between catastrophizing and pain. Clin J Pain. 2001;17(1):52-64.

38. Masselin-Dubois A, Attal N, Fletcher D, et al. Are psychological predictors of chronic postsurgical pain dependent on the surgical model? A comparison of total knee arthroplasty and breast surgery for cancer. J Pain. 2013;14(8):854-864.

39. Craner JR, Gilliam WP, Sperry JA. Rumination, magnification, and helplessness: how do different aspects of pain catastrophizing relate to pain severity and functioning? Clin J Pain. 2016;32(12): 1028-1035.

40. Schoenfeld-Smith K, Petroski GF, Hewett JE, et al. A biopsychosocial model of disability in rheumatoid arthritis. Arthritis Care Res. 1996;9(5):368-375.

41. Parker JC, Smarr KL, Angelone EO, et al. Psychological factors, immunologic activation, and disease activity in rheumatoid arthritis. Arthritis Care Res. 1992;5(4):196-201.

42. Boyden SD, Hossain IN, Wohlfahrt A, Lee YC. Non-inflammatory causes of pain in patients with rheumatoid arthritis. Curr Rheumatol Rep. 2016;18(6):30.

43. Affleck G, Tennen H, Urrows S, Higgins P. Neuroticism and the painmood relation in rheumatoid arthritis: insights from a prospective daily study. J Consult Clin Psychol. 1992;60(1):119-126.

44. O’Donovan A, Hughes BM, Slavich GM, et al. Clinical anxiety, cortisol and interleukin-6: evidence for specificity in emotion-biology relationships. Brain Behav Immun. 2010;24(7):1074-1077.

45. Sturgeon JA, Zautra AJ. State and trait pain catastrophizing and emotional health in rheumatoid arthritis. Ann Behav Med. 2013;45(1):69-77.

46. Edwards RR, Fillingim RB. Effects of age on temporal summation and habituation of thermal pain: clinical relevance in healthy older and younger adults. J Pain. 2001;2(6):307-317.

47. Ge HY, Madeleine P, Arendt-Nielsen L. Gender differences in pain modulation evoked by repeated injections of glutamate into the human trapezius muscle. Pain. 2005;113(1-2):134-140.
48. De Jongh RF, Vissers KC, Meert TF, Booij LH, De Deyne CS, Heylen RJ. The role of interleukin-6 in nociception and pain. Anesth Analg. 2003;96(4):1096-1103

49. Beilin B, Bessler H, Mayburd E, et al. Effects of preemptive analgesia on pain and cytokine production in the postoperative period. Anesthesiology. 2003;98(1):151-155.

50. Lee YC, Lu B, Bathon JM, et al. Pain sensitivity and pain reactivity in osteoarthritis. Arthritis Care Res (Hoboken). 2011;63(3):320-327.

51. Edwards RR, Wasan AD, Bingham CO 3rd, et al. Enhanced reactivity to pain in patients with rheumatoid arthritis. Arthritis Res Ther. 2009;11(3):R61.

52. Cleeland CS, Ryan KM. Pain assessment: global use of the brief pain inventory. Ann Acad Med Singapore. 1994;23(2):129-138.

53. Sullivan M J L BS, Pivik J. The pain catastrophizing scale: development and validation. Psychological Assessment 1995;7:524-532.

54. Beck AT, Ward CH, Mendelson M, Mock J, Erbaugh J. An inventory for measuring depression. Arch Gen Psychiatry. 1961;4:561-571.

55. Edwards RR, Mensing G, Cahalan C, et al. Alteration in pain modulation in women with persistent pain after lumpectomy: influence of catastrophizing. J Pain Symptom Manage. 2013;46(1):30-42.

56. Edwards RR, Wasan AD, Michna E, Greenbaum S, Ross E, Jamison RN. Elevated pain sensitivity in chronic pain patients at risk for opioid misuse. J Pain. 2011;12(9):953-963.

57. Peugh JL. A practical guide to multilevel modeling. J Sch Psychol. 2010;48(1):85-112.

58. Singer JD, Willett JB. Applied Longitudinal Data Analysis: Modeling Change and Event Occurrence. Oxford; New York: Oxford University Press; 2003.

59. Snijders TAB, Bosker RJ. Multilevel Analysis: An Introduction to Basic and Advanced Multilevel Modeling. London; Thousand Oaks, Calif: Sage Publications; 1999.

60. West BT, Welch KB, Galecki AT. Linear Mixed Models: A Practical Guide using Statistical Software. Boca Raton: Chapman \& Hall/CRC; 2007.

61. Friedman EM, Hayney M, Love GD, Singer BH, Ryff CD. Plasma interleukin-6 and soluble IL-6 receptors are associated with psychological well-being in aging women. Health Psychol. 2007;26(3):305-313.

62. Chatkoff DK, Leonard MT, Maier KJ. Pain catastrophizing differs between and within West Haven-Yale Multidimensonal Pain Inventory (MPI) pain adjustment classifications: theoretical and clinical implications from preliminary data. Clin J Pain. 2015;31(4):349-354.

63. Iwaki R, Arimura T, Jensen MP, et al. Global catastrophizing vs catastrophizing subdomains: assessment and associations with patient functioning. Pain Med. 2012;13(5):677-687.

64. Cheng J, Edwards LJ, Maldonado-Molina MM, Komro KA, Muller KE. Real longitudinal data analysis for real people: building a good enough mixed model. Stat Med. 2010;29(4):504-520.

65. Enders CK, Tofighi, D. Centering predictor variables in cross-sectional multilevel models: a new look at an old issue Psychol Methods 2007;12: 121-138.

66. Nezlek J. Multilevel random coefficient analyses of event and interval contingent data in social and personality psychology research, Psychological Bulletin 2001;27:771-785.

67. Kopala-Sibley DC, Zuroff DC, Russell JJ, Moskowitz DS, Paris J. Understanding heterogeneity in borderline personality disorder: differences in affective reactivity explained by the traits of dependency and self-criticism. J Abnorm Psychol. 2012;121(3):680-691.

68. Russell JJ, Moskowitz DS, Zuroff DC, Bleau P, Pinard G, Young SN. Anxiety, emotional security and the interpersonal behavior of individuals with social anxiety disorder. Psychol Med. 2011;41(3):545-554.

69. Wallace D, Green, B.S. Analysis of repeated measures designs with linear mixed models. In: Moscovitz DM, Hershberger SL, editors. Modeling Intraindividual Variability with Repeated Measures Data. NJ: Lawrence Erlbaum, Mahwah; 2002.

70. Schwartz JE, Stone AA. Strategies for analyzing ecological momentary assessment data. Health Psychol. 1998;17(1):6-16. 
71. Singer JD, Willett, J.B. Applied Longitudinal Data Analysis: Modeling Change and Event Occurrence. New York: Oxford University Press, Oxford; 2003.

72. Singer JD. Using SAS PROC MIXED to fit multilevel models, hierarchical models, and individual growth models. J Edu Behav Statistics 1998;23:323-325.

73. Brown CA, El-Deredy W, Jones AK. When the brain expects pain: common neural responses to pain anticipation are related to clinical pain and distress in fibromyalgia and osteoarthritis. Eur J Neurosci. 2014;39(4):663-672.

74. Brummett CM, Urquhart AG, Hassett AL, et al. Characteristics of fibromyalgia independently predict poorer long-term analgesic outcomes following total knee and hip arthroplasty. Arthritis Rheumatol. 2015;67(5):1386-1394.

75. Martel MO, Finan PH, Dolman AJ, et al. Self-reports of medication side effects and pain-related activity interference in patients with chronic pain: a longitudinal cohort study. Pain. 2015;156(6):1092-1100.

76. Lee YC, Lu B, Bathon JM, et al. Pain sensitivity and pain reactivity in osteoarthritis. Arthritis Care Res (Hoboken). 2011;63(3):320-327.

77. al'Absi M, Wittmers LE, Ellestad D, et al. Sex differences in pain and hypothalamic-pituitary-adrenocortical responses to opioid blockade. Psychosom Med. 2004;66(2):198-206.

78. Lutgendorf SK, Logan H, Costanzo E, Lubaroff D. Effects of acute stress, relaxation, and a neurogenic inflammatory stimulus on interleukin-6 in humans. Brain Behav Immun. 2004;18(1):55-64.

79. Edlinger C, Schreiber C, Goebel B, et al. Impact of Moderate Altitude on Pro-Inflammatory Cytokines in Healthy Volunteers. Clin Lab. 2017;63(9):1545-1548.

80. Haack M, Kraus T, Schuld A, Dalal M, Koethe D, Pollmacher T. Diurnal variations of interleukin-6 plasma levels are confounded by blood drawing procedures. Psychoneuroendocrinology. 2002;27(8):921-931.

81. Copeland S, Warren HS, Lowry SF, et al. Acute inflammatory response to endotoxin in mice and humans. Clin Diagn Lab Immunol. 2005;12(1):60-67.

82. Penninx BW, Abbas H, Ambrosius W, et al. Inflammatory markers and physical function among older adults with knee osteoarthritis. J Rheumatol. 2004;31(10):2027-2031.

83. Wallace DJ, Linker-Israeli M, Hallegua D, Silverman S, Silver D, Weisman MH. Cytokines play an aetiopathogenetic role in fibromyalgia: a hypothesis and pilot study. Rheumatology (Oxford). 2001;40(7):743-749.

84. Evers AW, Kraaimaat FW, Geenen R, Jacobs JW, Bijlsma JW. Stressvulnerability factors as long-term predictors of disease activity in early rheumatoid arthritis. J Psychosom Res. 2003;55(4):293-302.

85. Parker JC, Smarr KL, Walker SE, et al. Biopsychosocial parameters of disease activity in rheumatoid arthritis. Arthritis Care Res. 1991;4(2):73-80.
86. Zautra AJ, Burleson MH, Matt KS, Roth S, Burrows L. Interpersonal stress, depression, and disease activity in rheumatoid arthritis and osteoarthritis patients. Health Psychol. 1994;13(2):139-148.

87. Filiano AJ, Gadani SP, Kipnis J. How and why do T cells and their derived cytokines affect the injured and healthy brain? Nat Rev Neurosci. 2017;18(6):375-384.

88. Buenaver LF, Edwards RR, Haythornthwaite JA. Pain-related catastrophizing and perceived social responses: Inter-relationships in the context of chronic pain. Pain. 2007;127(3):234-242.

89. Christidis N, Ghafouri B, Larsson A, et al. Comparison of the levels of pro-inflammatory cytokines released in the vastus lateralis muscle of patients with fibromyalgia and healthy controls during contractions of the quadriceps muscle-A Microdialysis Study. PLoS One. 2015;10(12):e0143856.

90. Camacho EM, Verstappen SM, Chipping J, Symmons DP. Learned helplessness predicts functional disability, pain and fatigue in patients with recent-onset inflammatory polyarthritis. Rheumatology (Oxford). 2013;52(7):1233-1238.

91. Maes M, Song C, Lin A, et al. The effects of psychological stress on humans: increased production of pro-inflammatory cytokines and a Th1like response in stress-induced anxiety. Cytokine. 1998;10(4):313-318.

92. Kiecolt-Glaser JK, Preacher KJ, MacCallum RC, Atkinson C, Malarkey WB, Glaser R. Chronic stress and age-related increases in the proinflammatory cytokine IL-6. Proc Natl Acad Sci U S A. 2003;100(15):9090-9095.

93. Dantzer R, O’Connor JC, Freund GG, Johnson RW, Kelley KW. From inflammation to sickness and depression: when the immune system subjugates the brain. Nat Rev Neurosci. 2008;9(1):46-56.

94. Irwin MR, Miller AH. Depressive disorders and immunity: 20 years of progress and discovery. Brain Behav Immun. 2007;21(4):374-383.

95. Chandrashekara S, Jayashree K, Veeranna HB, et al. Effects of anxiety on TNF-alpha levels during psychological stress. J Psychosom Res. 2007;63(1):65-69.

96. Anisman H, Merali Z, Poulter MO, Hayley S. Cytokines as a precipitant of depressive illness: animal and human studies. Curr Pharm Des. 2005;11(8):963-972.

97. Schiepers OJ, Wichers MC, Maes M. Cytokines and major depression. Prog Neuropsychopharmacol Biol Psychiatry. 2005;29(2):201-217.

98. Manning PJ, Sutherland WH, McGrath MM, de Jong SA, Walker RJ, Williams MJ. Postprandial cytokine concentrations and meal composition in obese and lean women. Obesity (Silver Spring). 2008;16(9):2046-2052.

99. Silverman HA, Dancho M, Regnier-Golanov A, et al. Brain regionspecific alterations in the gene expression of cytokines, immune cell markers and cholinergic system components during peripheral endotoxin-induced inflammation. Mol Med. 2015;20:601-611.

\section{Journal of Pain Research}

\section{Publish your work in this journal}

The Journal of Pain Research is an international, peer reviewed, open access, online journal that welcomes laboratory and clinical findings in the fields of pain research and the prevention and management of pain. Original research, reviews, symposium reports, hypothesis formation and commentaries are all considered for publication.
The manuscript management system is completely online and includes a very quick and fair peer-review system, which is all easy to use. Visit http://www.dovepress.com/testimonials.php to read real quotes from published authors. 